\title{
SELF-ADJOINT EXTENSIONS OF THE TWO-VALLEY DIRAC OPERATOR WITH DISCONTINUOUS INFINITE MASS BOUNDARY CONDITIONS
}

\author{
Biagio CASSANO AND Vladimir LOTOREICHIK
}

\begin{abstract}
We consider the four-component two-valley Dirac operator on a wedge in $\mathbb{R}^{2}$ with infinite mass boundary conditions, which enjoy a flip at the vertex. We show that it has deficiency indices $(1,1)$ and we parametrize all its self-adjoint extensions, relying on the fact that the underlying two-component Dirac operator is symmetric with deficiency indices $(0,1)$. The respective defect element is computed explicitly. We observe that there exists no self-adjoint extension, which can be decomposed into an orthogonal sum of two two-component operators. In physics, this effect is called mixing the valleys.
\end{abstract}

Mathematics subject classification (2010): 35P05, 35Q40, 81Q10.

Keywords and phrases: Dirac operator, infinite mass boundary condition, wedge, self-adjoint extensions, mixing the valleys.

\section{REFERENCES}

[1] N. Arrizabalaga, L. Le Treust and N. Raymond, On the Mit bag model in the nonrelativistic limit, Comm. Math. Phys. 354 (2017), 641-669.

[2] N. Arrizabalaga, A. Mas and L. Vega, Shell interactions for Dirac operators, J. Math. Pures Appl. 102 (2014), 617-639.

[3] J. Behrndt, P. Exner, M. Holzmann And V. Lotoreichik, On the spectral properties of Dirac operators with electrostatic $\delta$-shell interactions, J. Math. Pures Appl. 111 (2018), 47-78.

[4] J. Behrndt, P. Exner, M. Holzmann and V. Lotoreichik, On Dirac operators in $\mathbb{R}^{3}$ with electrostatic and Lorentz, scalar $\delta$-shell interactions, Quantum Stud. Math. Found. 6 (2019), 295-314.

[5] J. Behrndt, M. Holzmann, T. Ourmières-Bonafos and K. Pankrashkin, Twodimensional Dirac operators with singular interactions supported on closed curves, J. Funct. Anal. 279 (2020), 108700.

[6] B. CASSANO AND F. Pizzichillo, Self-adjoint extensions for the Dirac operator with Coulomb-type spherically symmetric potentials, Lett. Math. Phys. 108 (2018), 2635-2667.

[7] B. Cassano And F. Pizzichillo, Boundary triples for the Dirac operator with Coulomb-type spherically symmetric perturbations, J. Math. Phys. 60 (2019), 041502.

[8] P. EXNER, Momentum operators on graphs, in "Spectral Analysis, Differential Equations and Mathematical Physics: A Festschrift in Honor of Fritz Gesztesy's 60th Birthday" (H. Holden, B. Simon, G. Teschl, eds.), Proc. Symp. Pure Math., 87, AMS, Providence, R.I.; (2012) pp. 105-118.

[9] P. EXNER AND P. ŠEBA, A simple model of thin-film point contact in two and three dimensions, Czechoslovak J. Phys. B 38 (1988), 1095-1110.

[10] M. Holzmann, T. OURmières-Bonafos And K. Pankrashinin, Dirac operators with Lorentz scalar interactions, Rev. Math. Phys. 30 (2018), 1850013.

[11] L. Le Treust AND T. OURMiÈres-Bonafos, Self-adjointness of Dirac operators with infinite mass boundary conditions in sectors, Ann. Henri Poincaré 19 (2018), 1465-1487.

[12] M. MarlettA AND G. Rozenblum, A Laplace operator with boundary conditions singular at one point, J. Phys. A 42 (2009), 125204, 11 pp. 
[13] W. MCLean, Strongly Elliptic Systems and Boundary Integral Equations, Cambridge University Press, 2000.

[14] S. A. NAZARov AND N. PopofF, Self-adjoint and skew-symmetric extensions of the Laplacian with singular Robin boundary condition, C. R. Math. Acad. Sci. Paris 356 (2018), 927-932.

[15] A. C. Neto, F. Guinea, N. M. Peres, K. S. Novoselov And A. K. Geim, The electronic properties of graphene, Rev. Mod. Phys. 81 (2009), 109-162.

[16] T. OURmiÈres-BonAfos AND F. PiZZICHILlo, Dirac operators and shell interactions: a survey, to appear in Mathematical Challenges of Zero-Range Physics (Springer INdAM Series).

[17] T. OURMiÈres-BONAFOS AND L. VEGA, A strategy for self-adjointness of Dirac operators: applications to the MIT bag model and $\delta$-shell interactions, Publ. Mat. 62 (2018), 397-437.

[18] F. Pizzichillo and H. VAn Den Bosch, Self-Adjointness of two dimensional Dirac operators on corner domains, arXiv:1902.05010.

[19] M. ReED AND B. Simon, Methods of modern mathematical physics. vol. 2. Fourier analysis, Academic Press, New York, 1975.

[20] J. Tworzydeo, B. Trauzettel, M. Titov, A. Rycerz and C. W. J. Beenakker, SubPoissonian shot noise in graphene, Phys. Rev. Lett. 96 (2006), 246802. 